\title{
Análise do Ciclo de Vida (ACV) aplicada ao agronegócio - Uma revisão de literatura
}

Edison S. Claudino ${ }^{1}$ \& Edson Talamini ${ }^{2}$

\section{RESUMO}

A preocupação com a dimensão ambiental da sustentabilidade tem ganhado relevância em pesquisas científicas aplicadas no agronegócio. A necessidade de um framework robusto e confiável para mensuração dos impactos ambientais no agronegócio apresenta-se como tendência em países líderes na produção mundial de alimentos, como o Brasil. Neste contexto o presente artigo descreve uma revisão de literatura acerca do conceito de Análise de Ciclo de Vida - ACV, o qual está sendo amplamente utilizado e reconhecido por técnicos e pesquisadores mundiais para avaliação ambiental das cadeias de produção, permitindo várias aplicações nos sistemas produtivos. Este artigo foi elaborado a partir de uma pesquisa bibliográfica em artigos publicados em periódicos nacionais e internacionais, sítios da internet e anais de congressos cujo objetivo é descrever a importância da aplicação e difusão do framework da ACV e suas possíveis aplicações no agronegócio brasileiro. O estudo conclui que este framework ainda é pouco difundido no agronegócio interno e o número de pesquisas com esta temática é reduzido.

Palavras-chave: biodiversidade, água, solo, resíduos, gestão ambiental

\section{Life Cycle Assessment (LCA) applied to agribusiness - A review}

\begin{abstract}
The concern with the environmental dimension of sustainability has become more and more relevant in studies related to agribusiness. The need of a robust and trusty framework to measure the environmental impacts in the agribusiness activities is being presented as a trend in countries leaders in food production worldwide, like Brazil. In this context, this paper describes a review related to the concept of Life Cycle Assessment - LCA which is being widely recognized and used by technicians and researchers all over the world to the environmental impacts of production chains and allowing various applications on the production systems. The paper was elaborated based on a bibliographical research carried out by accessing national and international publications, websites and proceedings of scientific events. Its main goal is to describe the importance and the diffusion of LCA as a framework to analyse production systems in Brazilian agribusiness. In conclusion, the LCA framework is still not widespread in the Brazilian agribusiness as well as the studies about this subject are still very scarce.
\end{abstract}

Key words: biodiversity, fresh water, soils, waste, environmental management 


\section{INTRODUÇÃO}

O agronegócio tem sido de fundamental importância para a geração de riquezas e manutenção da economia brasileira, através da geração de empregos, equilíbrio das contas e balança comercial, substituição de importação e garantia de alimentos com preços baixos e de qualidade. Através do avanço tecnológico e da implementação de novas técnicas de produção o Brasil se posicionou como destaque para o suprimento de alimentos para o mundo. O país é reconhecido como líder na produção mundial de: suco de laranja, café, cana-de-açúcar, além de ser um dos maiores produtores mundiais de: soja, carne, milho, biocombustíveis e outros.

Apesar da consolidação do agronegócio brasileiro, desafios recentes têm impactado o agronegócio, destacando-se: i - a crescente preocupação da sociedade quanto à sustentabilidade ambiental, tema que vem ganhando importância nos debates técnicos e científicos, gerando a necessidade de criação de uma produção sustentável e "limpa" e ii - nota-se que o mercado mundial apresenta, como tendência, a requisição de rotulagem e certificações de produtos elaborados sob critérios ambientais como requisitos para importação e comercialização.

Ante tais cenários surge a premência da criação de frameworks robustos e confiáveis através dos quais se possa avaliar, de forma segura, os efeitos prejudiciais para o meio ambiente dos sistemas de produção do agronegócio (produção agrícola e agroindústria). Desta forma, tornam-se imprescindíveis novos conhecimentos para aplicação nas cadeias produtivas capazes de auxiliar na tomada de decisão e na melhoria dos processos de produção. $\mathrm{O}$ enquadramento do agronegócio brasileiro sobre critérios ambientais poderá gerar a conquista de novos mercados além de proporcionar vantagem competitiva dos produtos agrícolas brasileiros sobre outros concorrentes mundiais.

A metodologia de elaboração deste trabalho consiste em uma revisão de literatura com base em referências bibliográficas obtidas a partir de artigos publicados em periódicos nacionais e internacionais, sites da internet e anais de eventos científicos; também são destacados prováveis tópicos para pesquisas futuras com a abordagem de Análise do Ciclo de Vida - ACV regionalizada e pontos que ainda carecem de consenso e permanecem pouco desenvolvidos nas pesquisas já produzidas com esta metodologia.

O trabalho possui, como etapas: i - introdução; ii abordagem de ACV aplicada ao agronegócio; iii - descrição da aplicação das estruturas de ACV; iv - análise dos estudos dos impactos do agronegócio, gerados na biodiversidade, água e solo; v- estudos de gerenciamento de resíduos a partir do conceito de ACV; vi - revisão das pesquisas produzidas no Brasil com a temática abordada e vii - conclusões.

O objetivo da pesquisa consiste em destacar a importância da difusão e a aplicação do framework de $\mathrm{ACV}$ no agronegócio e suas possíveis aplicações no Brasil, com o propósito de contribuírem para a criação de novos conceitos de produção, comparação e mensuração de tecnologias ecologicamente sustentáveis. Buscou-se analisar a aplicação da ACV sobre a óptica da produção agroindustrial, dos sistemas alimentares, conservação da biodiversidade e recursos fundamentais necessários para a produção do agronegócio (solo e água) visto que o tema é emergente e de fundamental importância.

\section{Abordagem de ACV APLICADA AO AGRONEGÓCIO}

Os atuais sistemas para produção de alimentos requerem grandes quantidades de entradas de recursos, o que provoca diversos efeitos negativos para o meio ambiente, sendo esses sistemas configurados e otimizados para satisfazer as demandas econômicas e as necessidades de crescimento da população mundial. Destaca-se haver dificuldades na condução dos estudos da ACV de produtos alimentares haja vista que um estudo completo deve incluir produção agrícola, refino industrial, estoque e distribuição, embalagem, consumo e gerenciamento de resíduos. Em conjunto, todos compõem um amplo e complexo sistema. Outra dificuldade é que os estudos de ciclos de vida envolvem muitas áreas científicas (multidisciplinares) (Andersson et al., 1998).

A ACV é uma técnica para avaliar o desempenho ambiental de determinado produto incluindo a identificação e a quantificação da energia e das matérias-primas utilizadas no seu ciclo de fabricação. Também são analisadas as emissões para água, solo e ar decorrentes da produção, utilização e disposição final avaliando-se o impacto ambiental associado ao uso dos recursos naturais (energia e matérias), emissões de poluentes e identificação de oportunidades para melhorar o sistema de forma a otimizar o desempenho ambiental do produto (Queiroz \& Garcia, 2010). Além disto, a ACV pode ser conceituada como ferramenta para avaliar os efeitos ambientais de um produto, processo ou atividade ao longo do seu ciclo de vida ou duração, conhecido como análise "do berço ao túmulo" (Roy et al., 2009).

De acordo com a Associação Brasileira de Ciclo de Vida (ABCV, 2011) no Brasil o acrônimo ACV significa, usualmente, análise de ciclo de vida, mas a tradução do inglês "life cycle assessment" também admite o significado: avaliação de ciclo de vida e em alguns países europeus se usa o termo "ecobalance", ou "ecoequilíbrio". Basicamente, esta técnica faz uma análise ou compilação de dados de um sistema de produto cujo resultado é avaliado posteriormente. Portanto, a ACV é uma análise (analysis) ao detalhar os fluxos de um sistema de produto e é uma avaliação (assessment) ao interpretar os fluxos. No Brasil o termo análise teve maior difusão entre os grupos de pesquisa, razão pela qual será adotado neste artigo.

As agroindústrias brasileiras vêm utilizando a ACV para as análises das questões ecológicas relacionadas às diversas etapas de produção, consumo e destino final de produtos ou serviços industriais e agrícolas. $\mathrm{O}$ objetivo é melhorar a eficiência da produção, contribuir para o desenvolvimento socioeconômico e auxiliar na conservação ambiental. Essas medidas podem dar suporte aos agentes do agronegócio na tomada de decisão quanto à definição dos insumos e ao investimento em novas tecnologias voltadas para a preservação ambiental (Barbosa Júnior, 2008).

\section{APLICAÇ̃̃o DA eSTRUTURA DE ANÁLISE DE CICLO DE VIDA}

O conceito de ACV emergiu na década de 1960 e desde a década de 1970 vários esforços para desenvolver uma 
metodologia acerca deste conceito, vêm sendo adotados. A partir de 1990 a SETAC (Society of Environmental Toxicology and Chemistry) e a USEPA (US Environmental Protection Agency) propuseram workshops e diversos projetos para desenvolver e promover um consenso sobre um framework visando à condução da Análise de Inventário do Ciclo de Vida e Avaliação de Impacto. Esforços similares foram empreendidos pelo SETACEurope, outras organizações internacionais de padronização de normas (tais como a International Organization for Standardization - ISO) e praticantes de pesquisas com esta abordagem em todo o mundo (Roy et al., 2009). A Figura 1 demonstra o objetivo da pesquisa na ACV.

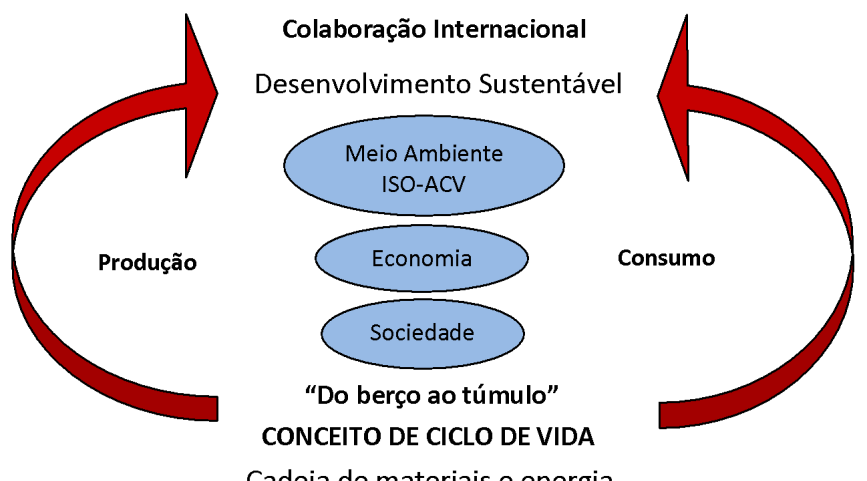

Cadeia de materiais e energia

Fonte: Adaptado do sítio do Instituto Nacional de Ciência Industrial Avançada e Tecnologia do Japão (AIST, 2011)

Figura 1. Objetivo da pesquisa na Análise do Ciclo de Vida (ACV)

A metodologia de uma ACV é estruturada nas quatro fases a seguir, de acordo com Roy et al. (2009):

- Definição dos objetivos e escopo: é uma das fases mais importantes visto que o estudo é realizado de acordo com o estabelecido nesta etapa, que define o propósito do estudo, o resultado esperado, os limites do sistema, unidade funcional (UF) e as suposições;

- Análise de inventário: é a fase mais trabalhosa e demorada comparada com outras fases, sobretudo pela coleta de dados; esta coleta pode consumir pouco tempo em caso de existirem boas bases de dados e se clientes e fornecedores estiverem dispostos a ajudar; informações de bases de dados podem ser utilizadas para processos que não são de produtos específicos, tais como dados gerais sobre a produção de eletricidade, carvão e embalagem;

- Avaliação de impacto: a Avaliação dos Impactos de Ciclo de Vida (AICV) visa compreender e avaliar os impactos ambientais com base na análise de inventário no âmbito da meta e no escopo do estudo, fase em que os resultados do inventário são atribuídos a diferentes categorias de impacto, com base nos tipos de impactos esperados ao meio ambiente; a Avaliação dos Impactos na ACV geralmente consiste dos seguintes elementos: classificação, caracterização, normatização e avaliação;

- Interpretação dos resultados: o propósito de uma ACV é se obter conclusões que possam apoiar uma decisão ou fornecer um resultado facilmente compreensível. O inventário e os resultados da avaliação de impacto são discutidos juntos, no caso de uma AICV, ou unicamente do inventário, no caso da análise ICV (Inventário de Ciclo de Vida) e significativas questões ambientais são identificadas para conclusão e recomendações compatíveis com os objetivos e escopo do estudo. As quatro fases previamente descritas podem ser visualizadas na Figura 2.

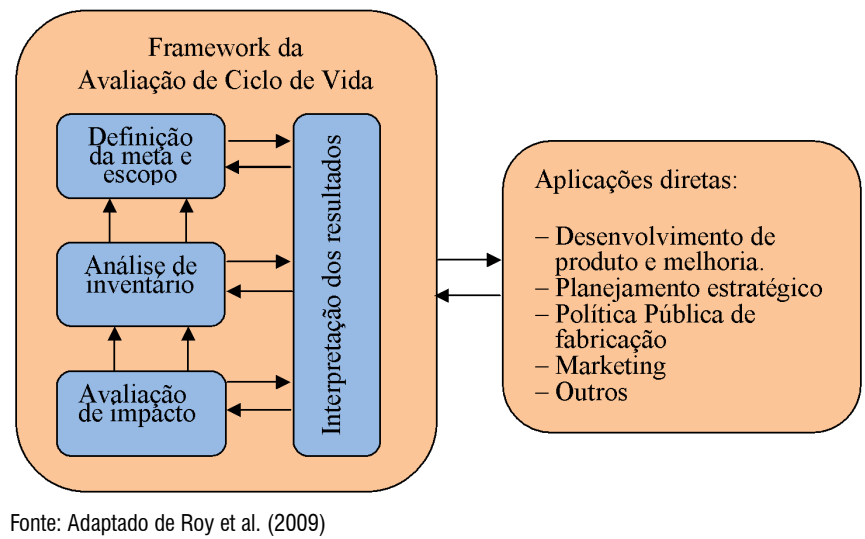

Figura 2. Estágio de uma Análise do Ciclo da Vida (ACV)

Desta forma, a ACV é uma metodologia interativa e sempre que os dados e as informações vão sendo compilados, os aspectos do escopo podem necessitar de modificações para se alcançar os objetivos originais do estudo (Eler \& Millani, 2007). Para aplicação dos estudos de ACV na produção de alimentos, Roy et al. (2009) destacam o crescimento da preocupação com a sustentabilidade da produção e consumo, solicitando diferentes atividades de pesquisa na produção e sistemas de produção, incluindo a produção agrícola.

No Brasil, a partir de 2009 a Associação Brasileira de Normas Técnicas (ABNT) publicou as versões em português das seguintes normas ISO (ABCV, 2011):

- ISO 14040 - Life Cycle Assessment: Principles and Framework (2006) - (Avaliação do Ciclo de Vida: Princípios e Estrutura).

- ISO 14044 - Life Cycle Assessment: Requirements and Guidelines (2006). (Avaliação do Ciclo de Vida: Requisitos e Orientações).

Apesar das normas de padronização para orientação e condução dos estudos em ACV, muitos indicadores propostos por pesquisadores e modelos utilizados em AICV para melhoramento e aperfeiçoamento em diferentes aspectos de uso do solo como mudança climática, biodiversidade, esgotamento de recursos e qualidade do solo, ainda não têm sido testados na prática nem comparados para aplicação (Mattila et al., 2012).

Em estudo elaborado por Thorn et al. (2011) para análise das emissões de gases nocivos gerados nas atividades industriais, os autores destacam pontos fracos associados com a validade e atualidade dos dados utilizados nos estudos de ACV em processos industriais. Para superá-los, os autores propõem duas ações fundamentais: i - a implantação de tecnologia que possa efetuar a compilação de dados das emissões gerados em tempo real para aumentar a acurácia e a validade na coleta de informações e análises elaboradas com base em modelos padronizados; e ii - divisão, em pequenas partes gerenciais, das emissões para análise da $\mathrm{ACV}$, podendo as melhorias na eficiência serem, então, levadas ao alcance de um grupo maior de empresas e à melhoria do controle sob seus fornecedores. 
Produtos agrícolas são tipicamente pouco agregadores de tecnologia para diferencial de mercado. Assim, a utilização de medidores na coleta de dados (ou seja, coleta de dados das emissões em caldeiras, processos térmicos, tratamentos químicos, cozimento, fermentação, destilação etc.) pode auxiliar na elaboração de políticas de "qualidade ambiental" dos produtos agroalimentares como vantagem competitiva e antecipação às futuras regulamentações e legislação voltadas para a redução dos efeitos das mudanças climáticas.

Predominantemente, a metodologia de ACV tem sido aplicada para produtos e processos industriais. Embora a maioria dos estudos de ciclo de vida realizados até agora envolva produção agrícola ou refino industrial, os vários estudos de ACV sobre produto agrícola têm incluído produção agrícola, processamento industrial, qualidade dos produtos alimentares acabados e a inclusão de etanol e biodiesel.

\section{ESTUDOS DOS IMPACTOS NA BIODIVERSIDADE, ÁGUA E SOLO UTILIZANDO-SE A FERRAMENTA DE ACV}

Por que estudar os impactos ambientais causados em decorrência das atividades do agronegócio? Ruviaro et al. (2012) destacam que na produção agrícola a aplicação de $\mathrm{ACV}$ é de grande relevância em todas as cadeias de produção o que pode ser observado pelos seguintes efeitos externos de mercado: $\mathrm{i}$ - consumidores exigem produtos que respeitam o meio ambiente e estão dispostos a pagar mais por eles; ii - os produtores que não são capazes de demonstrar que sua produção é cultivada de forma sustentável, têm dificuldades em acessar mercados importantes e iii - critérios ambientais estão sendo gradualmente adicionados pelos países para suas necessidades de importação de produtos agrícolas.

Os sistemas futuros para produção de bicombustíveis, alimentos e consumo, necessitam ter base global, visão ecológica, mínimo impacto ambiental e eficiente utilização dos recursos naturais, juntamente com importantes critérios no desenvolvimento de produtos alimentares e na seleção dos sistemas alimentares (Andersson, 2000).

\section{Estudos de ACV aplicados aos impactos na biodiversidade}

Atividades de produção agrícola exercem alto impacto sobre a biodiversidade do meio ambiente que a compõem. Importantes ferramentas e pesquisas têm sido desenvolvidas com a finalidade de se mensurar e avaliar esses impactos, o que é de fundamental importância para a sustentabilidade do agronegócio e para a melhoria do processo de produção. Os métodos de avaliação de impactos nas atividades agrícolas sobre a biodiversidade são uma relevante fonte de estudo para redução dos efeitos negativos da produção de alimentos sobre o meio ambiente e têm sido estudados por diversos pesquisadores que apontam novas ferramentas e métodos (Gaillard \& Nemecek, 2009).

Visando mensurar esses impactos, Lenzen et al. (2009) propõem um modelo baseado na construção de regressões múltiplas para mensuração entre espécies ameaçadas de extinção e o uso do solo, resultando em um amplo conjunto de coeficientes de correlação para ocupação do solo e ameaças a pássaros, mamíferos, plantas, répteis e anfíbios. Os coeficientes podem ser utilizados como aproximação entre as ameaças às espécies em extinção e apresentados em fatores prontos para serem testados em vários fatores de caracterização. Como resultado, os autores destacam que a legislação voltada à conservação da biodiversidade deve ir além das fronteiras nacionais dos países.

Jeanneret et al. (2008) também propõem um método desenvolvido para integração da biodiversidade como uma categoria de impacto da ACV para Produção Agrícola (SALCA-Biodiversity). Visando à realização da pesquisa os autores efetuaram três passos: i - criação de uma lista com 11 indicadores de grupos de espécie que foram estabelecidos considerando-se critérios de ACV e ecológicos; ii - dados do inventário acerca das práticas agrícolas de opções detalhadas de gerenciamento foram especificados e iii - um sistema de pontuação estimou a reação de todos os indicadores do grupo de espécie em relação às opções de gerenciamento, seguidos das etapas de agregação. O resultado do trabalho mostra a influência dominante da intensidade da produção, o gerenciamento na maioria dos indicadores e as opções de gerenciamento a partir da quais grandes impactos sobre a biodiversidade são esperados.

Outra preocupação relevante, comum em estudos, principalmente nos europeus realizados para a produção de combustíveis renováveis, aponta a problemática com o desmatamento de florestas para o uso do solo visando à implantação de programas de biodiesel em países tropicais. Alguns exemplos são as plantações de palma para a produção de óleo na Malásia, Indonésia, escudo guianês colombiano e Amazônia brasileira, além da produção de soja no extremo norte do Brasil, trazendo impacto para o uso do solo e para a biodiversidade (Renó et al., 2011; Monteiro, 2011).

Estudos de ACV aplicados com o propósito de identificar impactos na biodiversidade podem ser de grande importância para o Brasil haja vista que o País ainda possui áreas de floresta nativa e de preservação ambiental permanente a serem exploradas, inclusive com muitas áreas que podem combinar atividades de produção com a conservação de florestas.

No estado do Mato Grosso do Sul se destacam duas importantes atividades econômicas na Região do Pantanal Sul mato-grossense as quais possuem grande concentração de biodiversidade, como: a criação de gado bovino e a exploração de atividades turísticas através do ecoturismo, compatibilizando produção e sustentabilidade da natureza. Com isto, o desenvolvimento de ACV com abordagem regionalizada para analisar os impactos sob este bioma natural é de fundamental importância tornando-se, sem dúvida, uma fonte de estudo potencial a ser explorada em pesquisas empíricas.

\section{Estudos de ACV aplicados aos impactos sobre água doce}

Água é um recurso precioso e cada vez mais escasso. Isto é crítico para as funções do ecossistema (tanto como habitat e recurso) e igualmente essencial para os seres humanos. A água captada para propósitos humanos e produção agrícola pode ter significantes impactos sobre os sistemas e as espécies que sobrevivem na água doce.

Apesar da importância da água doce, os impactos sobre seu uso têm sido pouco representados desde o início da metodologia de ACV. Provavelmente, porque esta metodologia 
foi inicialmente desenvolvida para os sistemas industriais (geralmente menos dependentes de recursos hídricos do que a agricultura) em países abundantes em água.

Em geral, os estudos de ACV relatam a quantidade total de água usada pelo sistema de produção, "do berço" (aquisição de matéria-prima) "até o túmulo" (gerenciamento de resíduos). Em geral, tais estudos nem sequer distinguem as fontes a partir das quais a água é obtida nem a forma ou condição em que a água deixa o sistema de produção (Gaillard \& Nemecek, 2009).

Milà I Canals et al. (2009) descrevem como o uso da água pode ser abordado em ACV tendo como características as principais vias de impacto do suprimento sendo identificadas, levando à definição dos fluxos que requerem quantificação no ICV (Inventário de Ciclo de Vida).

$\mathrm{Na}$ definição dos autores duas terminologias abordadas para o uso da água doce sobre conceito de entrada e saída do sistema de produção, são:

- Conceito de entrada (origem) de água: a água ocorre na forma de "água verde" (que é armazenada como composto do solo e disponível para evaporação através das lavouras e vegetação terrestre) e "água azul" (armazenada na superfície ou águas subterrâneas).

- Conceito de saída (retorno para a natureza): i - uso da água sem evaporação onde a água é retornada para a base original e pode ser usada por outros usuários após a saída do sistema; ii - uso da água com evaporação onde a água é dissipada e não disponível imediatamente após o uso.

Os autores sugerem dois principais indicadores para as vias de impacto, que são:

- Impactos sobre o ecossistema de água doce (Freshwater Ecosystem Impact - FEI): indicadores relativos ao atual uso da água doce para a disposição dos recursos da água doce (com ou sem análises específicas das necessidades do ecossistema) são sugeridos.

- Esgotamento de água doce (Freshwater Depletion - FD): os parâmetros necessários para a avaliação comumente utilizada dos potenciais esgotamentos abióticos são explorados.

Como conclusão os autores apontam que ainda é necessária uma melhoria na representação dos impactos associados com o uso de água doce em ACV. O uso generalizado da abordagem sugerida pelos autores requer algum desenvolvimento (e consenso) para a criação de uma base de dados em ICV.

O Estado do Mato Grosso do Sul possui abundante quantidade de água doce, sendo banhado por duas importantes bacias hidrográficas: Bacia do Rio Paraguai e Bacia do Rio Paraná que, juntamente com seus afluentes, são de fundamental importância para algumas cadeias de produção, especialmente a piscicultura (criação de peixes de água doce) que se tem desenvolvido na Região da Grande Dourados (região abrangida pela cidade de Dourados e cidades limítrofes) e atividade do turismo de pesca em rios de água doce na Bacia do Rio Paraguai na Região do Pantanal Sul Mato-grossense.

A grande quantidade de água doce e "limpa" na região tem propiciado condições ímpares para o desenvolvimento de cadeias que carecem deste recurso. Tal condição gera oportunidades para o desenvolvimento de pesquisa de ACV a partir de uma abordagem local dos impactos desses sistemas produtivos (turismo de pesca e piscicultura) analisando critérios de sustentabilidade e impactos sobre os ecossistemas que sobrevivem na água.

\section{Estudos de ACV aplicados aos impactos sobre o solo}

Atividades humanas têm necessidades espaciais para extração de recursos, silvicultura e agricultura, infraestrutura e habitações, processos de produção industrial e aterro. Muitas vezes, o uso repetido torna o solo indisponível para outros fins mas pode também mudar a qualidade do solo em termos de suporte vital ou potencialidade para outros usos voltados à produção agrícola. Os impactos sobre o uso do solo têm sido pouco incluídos nos estudos de $\mathrm{ACV}$ devido à falta de uma metodologia estabelecida (Gaillard \& Nemecek, 2009).

Vários métodos têm sido desenvolvidos como tentativa para avaliação dos impactos ambientais gerados pelo uso do solo e mudanças decorrentes de sua exploração. Esses métodos e ferramentas ainda enfrentam problemas compartilhados e especíicos em relação à avaliação dos impactos. Entre esses problemas a seleção e a definição de indicadores mensuráveis e relevantes parecem ser as etapas mais difíceis. Discussões sobre os impactos do uso do solo nas pesquisas de $\mathrm{ACV}$ parecem revelar uma falta de consenso sobre o que exatamente tem que ser avaliado (Gaillard \& Nemecek, 2009).

Ruviaro et al. (2012) apontam que a metodologia de ACV apresenta pontos fracos por não considerar e/ou não incluir todos os impactos relevantes sobre a produção (o uso do solo e água, mudanças indiretas no uso do solo e a competição ecológica entre produtos). Também pela dificuldade para considerar a redução do uso do solo quando a produção é classificada como ecológica (orgânico, natural, biodinâmico, entre outros).

Lindeijer (2000) realizou uma revisão acerca das metodologias de impacto para uso do solo baseado no framework de ACV, destacando duas questões principais: i: como a ocupação de áreas se relaciona com (irreversíveis) mudanças do solo; e ii: quais indicadores devem ser utilizados para descrever impactos do uso do solo. A maioria das abordagens existentes visa a um único ou limitado número de índices para impactos do uso do solo devido à indisponibilidade de dados. Densidade de espécie de planta vascular é a base mais comum para um indicador. Para a silvicultura e lavouras agrícolas o autor recomenda que índices de multi-indicadores devem ser aplicados para comparar técnicas de gerenciamento de solo.

Considerando a falta de consenso sobre os indicadores a serem utilizados em uma metodologia para incorporar o uso do solo na ACV, Achten et al. (2008) propõem a criação de um conceito que descreve como é possível mensurar o funcionamento dos ecossistemas sem perdas para a biodiversidade e sustentabilidade na produção, a fim de compreender como o uso do solo pode afetá-las. Buscando identificar os pontos de impactos, os autores propõem um conjunto dinâmico de multi-indicadores para quantificação de pontos de médio impacto sobre a fertilidade do solo, biodiversidade, produção de biomassa, estrutura do solo, estrutura da vegetação e balanço hídrico local. Além dessas análises os autores apresentam um método de cálculo de impactos apropriado para diferentes ferramentas de avaliação 
ambiental e demonstram a incorporação na metodologia em ACV.

Mattsson et al. (2000) não só propõem o esboço de um método de avaliação ambiental para uso do solo agrícola mas definem objetivos ambientais e indicadores para qualidade do uso do solo. As subcategorias de impactos definidos na pesquisa são: fertilidade do solo, biodiversidade e valores paisagísticos.

Complementando a análise em subcategorias os autores ainda propõem sete indicadores para as variáveis quantificáveis de sustentabilidade da capacidade de produção do solo agrícola: a erosão do solo, hidrologia, matéria orgânica do solo, estrutura do solo, pH do solo, acumulação de metais pesados, nível de fósforo $(\mathrm{P})$ e potássio $(\mathrm{K})$ do solo. Além desses quantificadores a pesquisa realizou estudo de caso com três diferentes vegetais oleaginosos em três diferentes países: soja do Brasil, óleo de palma da Malásia e semente de colza da Suécia.

Como resultado da pesquisa os autores concluíram que esses indicadores fornecem boa imagem de longo prazo a respeito da fertilidade do solo e biodiversidade porém tomá-los juntos envolve resultados que são um composto de informações quantitativas e qualitativas, o que se torna difícil de agregar em um nível aceitável.

O cerrado brasileiro passou por um avanço considerável em sua fronteira agrícola e pecuária de corte, através do desenvolvimento de pesquisas, o que possibilitou também o aumento da produtividade em diversas culturas. Contudo, novos desafios surgem em pesquisas voltadas para a aplicação no uso dos solos devido ao crescimento da demanda por alimentos e proteína animal ocasionada pelo aumento das exportações para os países emergentes e a competição das áreas agricultáveis entre alimentos e biocombustíveis gerando forte pressão pelo aumento da produtividade.

Neste contexto se destacam, como futuras temáticas importantes de pesquisa em ACV aplicadas ao uso dos solos: i - necessidade de aumento da produção de alimentos (grãos) gerando aumento da degradação ocasionada pela exploração intensiva do fator terra (solo) e os efeitos sobre sua mudança no longo prazo e ii - produção de proteína animal, especialmente para produção de carne vermelha (bovina) que se utiliza de grandes extensões de terra com baixa produtividade, tornando-se oportuno o desenvolvimento de novas técnicas de conservação do solo para pastagens e a criação em espaços menores (confinamentos e menor prazo de abate).

\section{Gerenciamento DE RESÍDUOS A PARTIR DO CONCEITO DE ACV}

O desenvolvimento de novas tecnologias passíveis de produzir e elaborar sistemas e processos que possibilitem a reutilização de resíduos dos sistemas de produção agrícola, processos agroindustriais, energia, adubação, reciclagem e outros, é de fundamental importância para garantir a sustentabilidade da produção limpa nos sistemas e cadeias produtivas do agronegócio.

A forte dependência do uso intenso de combustíveis fósseis e derivados, combinada com a diminuição das reservas de petróleo, causa preocupações políticas e ambientais. A utilização dos resíduos da agricultura como matéria-prima em biorrefinaria é uma alternativa promissora para os recursos fósseis, para a produção de vetores de energia e para produtos químicos mitigando, assim, mudanças climáticas e melhorando a segurança energética.

A partir deste contexto Cherubini \& Ulgiati (2009) elaboraram um estudo referente o conceito de biorrefinaria, que produz bioetanol, bioenergia e bioquímicos com dois tipos diferentes de resíduos agrícolas: palha de milho e palha de trigo, utilizando a abordagem de ACV que leva em conta todos os fluxos de entrada e saída que ocorrem ao longo da cadeia de produção. As conclusões mostram que o uso de resíduos de colheitas em uma biorrefinaria poupa cerca de $50 \%$ de emissões de gases de efeito estufa (greenhouse gases - GH) e reduzem a demanda de energia fóssil em cerca de $80 \%$.

Com base nesses resultados constata-se, a partir de resíduos, que um conceito de biorrefinaria possibilita resolver dois problemas: encontrar um uso alternativo para os resíduos abundantes de lignocelulose e assegurar a mitigação dos efeitos negativos para a maioria das preocupações ambientais relacionadas aos recursos energéticos não renováveis.

A minimização de resíduos na indústria de alimentos tem levado a melhorias demonstradas em outros setores - eficiência energética, redução do uso de matérias-primas, redução do consumo de água e aumento do seu reúso e reciclagem. A geração de efluentes líquidos com elevado conteúdo orgânico e a geração de alta quantidade de lodo (material semissólido proveniente de efluentes) e resíduos sólidos são acatados como problemas comuns a todas as indústrias de alimentos e agroindústrias, em geral (Roy et al., 2009).

Os impactos ambientais da reciclagem, os mecanismos de tratamento biológico (Mechanical Biological Treatements MTB) e a incineração de resíduos para a geração de energia, são revisados e comparados por Valério (2010). No estudo são citadas as principais estratégias de gerenciamento para responder à crescente produção de materiais pós-consumo, em referência aos impactos ambientais. Estudos realizados com a abordagem de ACV confirmam que o menor impacto ambiental sobre uma escala global é obtido pela reciclagem e tratamento biológico (compostagem e fermentação anaeróbica) caso o composto seja usado na agricultura.

Os fatores disponíveis de emissões de gases sugerem que, em escala local, mecanismos de tratamento com recuperação de energia de biogás podem ser intrinsecamente mais seguros do que incineradores de resíduos para geração de energia. Como conclusão os estudos destacam que mais pesquisas são necessárias para comparar os impactos ambientais dos Mecanismos de Tratamento Biológicos (Mechanical Biological Treatments - MTB) e incineradores de resíduos para a geração de energia.

A geração de resíduos dos sistemas industriais, resíduos urbanos e resíduos dos sistemas agrícolas pode gerar graves efeitos a longo prazo e, em muitos casos, necessitar de complexos e caros processos de recomposição e reutilização para serem descartados ou reaproveitados no meio ambiente.

Pesquisas científicas se têm direcionado para importantes caminhos, sobremaneira para a reciclagem de resíduos agrícolas destinados à produção de biocombustíveis de segunda geração, os quais podem ter seu volume ampliado em todos os padrões 
que convertem resíduos lignocelulósicos em bioenergia e bioquímicos. Esta alternativa torna possível utilizar-se uma infinidade de plantas e resíduos agrícolas como matéria-prima além de muitos resíduos possibilitarem alternativas locais de reaproveitamento para a geração de biogás e incineração como fonte de energia.

Após o processo de reciclagem e tratamento biológico (compostagem e fermentação anaeróbica) diversos resíduos podem ser utilizados na agricultura para adubação e alcançar importantes resultados ambientais auxiliando na produção e na recomposição de nutrientes para a produção agrícola.

Aperfeiçoar pesquisas para otimização e reutilização de recursos é de fundamental importância, principalmente aquelas baseadas no framework de ACV as quais podem mapear e identificar os pontos de maior utilização de energia, geração de resíduos e impactos ambientais. Avanços nesta direção podem proporcionar a oportunidade de elaboração de políticas públicas e melhoramento nos processos de produção para redução nos danos ao meio ambiente, além de alternativa para a geração de energia e calor em agroindústrias.

\section{APLICAÇÃO DO CONCEITO DE ACV EM ESTUDOS NO BRASIL}

No Brasil, os estudos de ACV ainda são incipientes e raros são dados disponíveis acerca da avaliação de impacto no País e bases de dados a serem refinadas. É importante a realização de trabalhos locais pois como a metodologia de ACV surgiu na Europa, muitas das informações e aplicações nos estudos de casos e testes empíricos devem ser analisadas com uma abordagem regionalizada devido às características particulares do Brasil, como: condições climáticas, fatores de produção, sistemas produtivos, sistemas de gerenciamento, reciclagem de resíduos, etc.

Ruviaro et al. (2012) destacam a necessidade urgente de mais pesquisas de ACV voltadas para produtos agrícolas de vez que a maioria das pesquisas de ACV elaboradas internamente, país tem, como análise, grãos, vegetais e outros. A Figura 3 compara a distribuição dos estudos de ACV de acordo com o tipo de produto agrícola no Brasil e no Mundo.

Neste contexto, uma breve revisão dos principais trabalhos da ACV elaborados no Brasil com características locais e suas cadeias de análise, é apresentada na sequência.

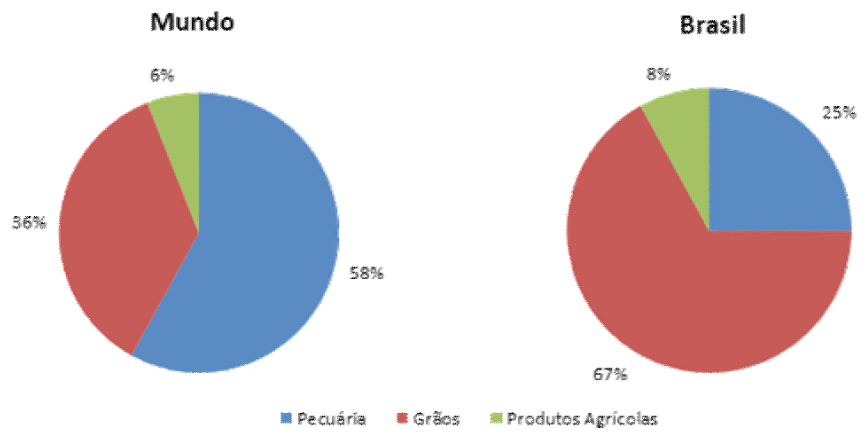

Fonte: Adaptado de Ruviaro et al. (2012)

Figura 3. Distribuição dos diferentes tipos de estudo sobre ACV de acordo com o tipo de produto agrícola (Mundo e Brasil)
Coltro et al. (2008) pesquisaram a cadeia de produção de suco de laranja concentrado e congelado produzido no estado de São Paulo, analisando a quantidade de energia utilizada no seu ciclo de vida. A pesquisa teve como meta apresentar o uso de energia em todas as etapas do clico de vida e, como escopo, qualificar e quantificar os principais aspectos ambientais. Os resultados mostram que aproximadamente $70 \%$ do uso de energia são destinados para o cultivo da laranja uma vez que a proporção de laranja para a produção do suco de laranja concentrado e congelado é de 10:1.

Na produção de café verde, da qual o Brasil é um dos grandes exportadores mundiais e possui grande consumo interno, Coltro et al. (2006) realizaram um estudo de ICV com produtores de café de 04 regiões: Cerrado Mineiro, Sul do estado de Minas Gerais, as regiões da Alta Mogiana e Marília no centro-oeste do estado de São Paulo, visando realizar um perfil ambiental do café verde nacional para obter dados detalhados de inventário da produção. O trabalho teve como resultado o fornecimento de uma correlação melhor das práticas agrícolas e os potencias impactos ambientais gerados por esta cadeia de produção.

No setor de produção de aves para corte, que obteve crescimento recente no País, Silva et al. (2008) comparam as duas atuais cadeias de produção de ave no Brasil: o Sistema do Sul interna e o Sistema de produção do Centro-Oeste. O estudo mostrou que a distância de transporte é o fator predominante para a avaliação e grande parte da contribuição dos impactos ambientais provém da produção de alimentos para as aves que inclui a produção de ração de milho e soja e seu transporte até os pontos de produção e consumo. Analisando todas as etapas de produção, o estudo conclui que o sistema de produção localizado no Centro Oeste é mais vantajoso para as categorias de emissão de gases de efeito estufa e recursos energéticos.

$\mathrm{Na}$ análise da Cadeia da Soja, que é altamente dependente da entrada de insumos para produção, como: solo, fertilizantes, combustíveis máquinas e outros, Silva et al. (2010) analisaram a variabilidade dos impactos ambientais da soja brasileira, de acordo com a produção agrícola e o cenário de transporte. O objetivo do estudo foi avaliar os impactos ambientais das cadeias de fornecimentos nas duas maiores regiões produtores de soja do Brasil: Região Sul e Região Centro-Oeste. Os autores concluíram que a escolha do modo de transporte e a distância a ser percorrida influenciam bastante os impactos ambientais.

O Brasil, além de ser um dos pioneiros no desenvolvimento de biocombustíveis é, atualmente, um dos grandes produtores mundiais e tem apresentado pesquisas relevantes baseadas no framework de ACV para combustíveis renováveis e subprodutos agrícolas como alternativas para a geração de energia e calor.

Neste contexto, Ometto \& Roma (2010) elaboraram um estudo significativo na cadeia sucroalcooleira acerca da produção de etanol associada à cogeração de eletricidade e concluíram que a atividade de maior emissão de gás carbônico para a atmosfera proveniente desta atividade é devida à colheita da cana, em virtude do metano emitido por ocasião da queima pré-colheita. Como melhoria da produção os autores propõem a realização da colheita da cana-de-açúcar sem queima, a utilização de combustíveis renováveis em tratores, caminhões e ônibus, ao invés de combustíveis fósseis, e a otimização da logística de transporte de produtos e insumos. 
Garcia \& Sperling (2010) também pesquisaram a cadeia sucroalcooleira analisando as estimativas de emissões de gases de efeito estufa $\left(\mathrm{CO}_{2}, \mathrm{CH}_{4}, \mathrm{~N}_{2} \mathrm{O}\right)$ nas etapas agrícolas e industrialização da cana-de-açúcar no estado de Minas Gerais, concluindo que a maior parte das emissões é proporcionada pela queima da cana-de-açúcar e pelo consumo de combustíveis.

Considerando a necessidade de analisar as consequências negativas dos programas de biocombustíveis, como aqueles relacionados à competição com alimentos e sustentabilidade, Renó et al. (2011) buscaram contribuir para o desenvolvimento de um framework para indicadores de sustentabilidade como ferramenta para avaliação de desempenho. Na pesquisa, os autores compararam vários indicadores enfatizando suas vantagens e desvantagens. As principais limitações relacionadas pelas fronteiras estudadas, bem como a falta de dados confiáveis e seus efeitos, são discutidos com base em estudos de casos de Ciclo de Vida reais realizados pelos autores acerca do biodiesel de óleo de palma e diferentes alternativas para tratamento de vinhaça e descarte de resíduos.

Analisando os efeitos da produção e a industrialização da cana-de-açúcar, que é atualmente a principal fonte de energia renovável no Brasil e de grande importância na agroindústria, Seabra et al. (2011) realizaram estudo com o objetivo de analisar o ciclo de vida do uso de energia e emissões de gás carbônico relacionados à cana-de-açúcar e etanol considerando também os excedentes de eletricidade e bagaço como subprodutos. Os pesquisadores realizaram um balanço completo para a Região Centro-Sul brasileira, adotando diferentes métodos para avaliar, separadamente, a produção de etanol e cana-de-açúcar; como conclusão a pesquisa aponta que os produtos derivados da cana-de-açúcar são mais vantajosos quando comparados com o açúcar de beterraba produzido na Europa.

Visando à criação de frameworks para análises ambientais confiáveis para os processos produtivos, Alvarenga et al. (2012) comparam o método da Pegada Ecológica (Ecological Footprint - EF) com o framework de ACV Os autores utilizaram estudo de caso sobre a produção de ração para frango de corte e obtiveram, como conclusão, que a Pegada Ecológica não é adequada para o setor agrícola haja vista que decisões enganosas podem ser tomadas como resultado da negligência de alguns importantes impactos no sector econômico.

Para realização de Inventário de Ciclo de Vida - ICV, Alvarenga et al. (2008) pesquisaram o sistema de produção de ostras brasileiras tendo, como meta, o levantamento de dados de entrada e saída em todos os estágios do Ciclo de Vida para a produção de ostras visando fornecer fundamentos para futuras análises de ACV. As conclusões da pesquisa apresentam a identificação no sistema de produção do alto consumo de água (água doce e salgada), altas emissões de Dióxido de Carbono $\left(\mathrm{CO}_{2}\right)$, alta quantidade total de resíduos sólidos (em águas residuais) e resíduos sólidos como casca de ostras.

\section{Conclusões}

1. No Brasil ainda se verificam poucas pesquisas com a abordagem em Análise do Ciclo da Vida (ACV); no entanto, o tema é emergente e vem ganhando espaço em razão da crescente preocupação da sociedade quanto às práticas produtivas sustentáveis, socialmente justas e economicamente viáveis.

2. Nota-se a falta de conhecimento da metodologia de ACV para pesquisas em universidades e empresas voltadas para o agronegócio, além de dificuldades para a realização de pesquisas com esta abordagem em virtude da falta de recursos financeiros para execução de projetos e da necessidade de uma abordagem multidisciplinar.

3. Destaca-se a importância da difusão de pesquisas com o framework de ACV em universidades, agroindústrias em posições estratégicas em suas cadeias produtivas e na EMBRAPA, para uso como ferramenta estratégica cujas aplicações são passíveis de gerar vantagem competitiva para acesso a novos mercados internacionais e auxiliar nas certificações ambientais internacionais e sanitárias para exportações.

4. Ressalta-se a necessidade de esforços com vista à melhoria da metodologia de ACV, em normas específicas e métodos amplamente reconhecidos para avaliação do uso de solo, impactos sobre a biodiversidade, recursos hídricos e impactos de defensivos agrícolas que ainda estão incompletos, o que limita a validade dos resultados de aplicação da ACV.

\section{LITERATURA CITADA}

ABCV - Associação Brasileira de Ciclo de Vida. O conceito de ciclo de vida e definição de ACV. http://www.abcvbrasil. org.br/index.php. 15 Ago. 2011.

Achten, W. M. J.; Mathijs, E.; Muys, B. Proposing a life cycle land use impact calculation methodology. In: International Conference on LCA in the Agri-Food Sector, 6, 2008, Zurich. Proceedings...Zurich: Agroscope ReckenholzTänikon Research Station ART, 2008. p.22-33.

AIST - National Institute of Advanced Industrial Science and Technology. Research Center for Life Cycle Assessment Research theme. http://www.aist-riss.jp/old/lca/cie/theme/ index.html. 18 Ago. 2011

Alvarenga, R. A. F.; Silva, V. S. R. P. da. Comparison of the ecological footprint and a life cycle impact assessment method for a case study on Brazilian broiler feed production. Journal of Cleaner Production, v.28, p.25-32, 2012.

Alvarenga, R. A. F.; Soares S. R.; Silva, V. S. R. P. Life cycle inventory for a Brazilian oyster production system. In: International conference on LCA in the agri-food sector, 6, 2008. Zurich. Book of Abstracts...Zurich: Agroscope Reckenholz-Tänikon Research Station ART, 2008, p.102.

Andersson, K. LCA of food products and production systems. International Journal of Life Cycle Assessment, v.5, p.239248, 2000.

Andersson, K; Ohlsson, T.; Olsson, P.; Screening life cycle assessment (LCA) of tomato ketchup: A case study. Journal of Cleaner Production, v.6, p.277-288, 1998.

Barbosa Júnior, A. F. Conceitos e aplicações de análise do ciclo de vida (ACV) no Brasil. Revista Gerenciais, v.7, p.39-44, 2008.

Cherubini, F.; Ulgiati, S. Crop residues as raw materials for biorefinery systems - A LCA case study. Appl Energy, v.53, p.434-447, 2009. 
Coltro, L.; Mourad, A. L.; Germer, S. P. M.; Mendonça, T. A.; Kletecke, R. M. Energy use in the life cycle of frozen concentrated orange juice produced in Brazil. In: International conference on LCA in the agri-food sector, 6 , 2008, Zurich. Proceedings...Zurich: Agroscope ReckenholzTänikon Research Station ART, 2008. p.228-233.

Coltro, L.; Mourad, A. L; Oliveira, P. A. P. L. V. ; Baddini, J. P. O. A. ; Kletecke, R. M. Environmental profile of Brazilian green coffee. International Journal of Life Cycle Assessment, v.11, p.16-21, 2006.

Eler, M. N.; Millani, T. J. Métodos de estudos de sustentabilidade aplicados a aquicultura. Revista Brasileira de Zootecnia, v.36, p.33-44, 2007.

Garcia, J. C. C.; Sperling, E. von. Greenhouse gas emissions in the life cycle of ethanol: Estimation in agriculture and industrialization stages in Minas Gerais, Brazil. Engenharia Sanitária e Ambiental, v.15, p.217-222, 2010.

Jeanneret, P.; Baumgartner, D. U.; Knuchel R. F.; Gaillard, G. A new LCIA method for assessing impacts of agricultural activities on biodiversity (SALCA-Biodiversity). In: International Conference on LCA in the Agri-Food Sector, 6, 2008, Zurich. Proceedings...Zurich: Agroscope ReckenholzTänikon Research Station ART, 2008, p.34-39.

Lenzen, M.; Lane, A.; Widmer-Cooper, A.; Williams, M. Effects of land use on threatened species. Conservation Biology, v.23, 294-306, 2009.

Lindeijer, E. Review of land use impact methodologies. Journal of Cleaner Production, v.8, p.273-281, 2000.

Mattila, T.; Helin, T.; Antikainen, R. Land use indicators in life cycle assessment: A case study on beer production. International Journal of Life Cycle Assessment, v.12, p.277-286, 2012.

Mattson, B.; Cederberg, C.; Blix, L. Agricultural land use in life cycle assessment (LCA): Case studies of three vegetable oil crops. Journal of Cleaner Production, v.8, p.283-292, 2000.

Milà i Canals, L.; Chenoweth, J.; Chapagain, A.; Orr, S.; Antón, A.; Clift, R. Assessing freshwater use impacts in LCA: Part I - Inventory modelling and characterisation factors for the main impact pathways. International Journal of Life Cycle Assessment, v.14, p.28-42, 2009.

Monteiro, K. F. G. Contribuições para a gestão ambiental da cadeia produtiva de biodiesel na amazônia Brasileira e Colombiana. Revista Oecologia Australis, v.15, p.351-354, 2011.
Nemecek, T.; Gaillarb, G. Conference Report. Agri-Food $6^{\text {th }}$ International Conference on LCA in the Agri-Food Sector. International Journal of Life Cycle Assessement, v.17, p.687-689, 2009.

Ometto, A. R.; Roma, W. N. L. Atmospheric impacts of the life cycle emissions of fuel ethanol in Brazil: Based on chemical exergy. Journal of Cleaner Production, v.18, p.71-76, 2010.

Queiroz, G. C.; Garcia, E. E. C. Reciclagem de sacolas plásticas de polietileno em termos de inventário de ciclo de vida. Revista Polimeros, v.20, p.401-406, 2010.

Renó, M. L. G.; Lora, E. E. S.; Palacio, J. C. E; Rocha, M. H.; Venturini, O. J.; Olmo, O. A. Issues to consider, existing tools and constraints in biofuels sustainability assessments. Energy, v.36, p.2097-2110, 2011.

Roy, P.; Nei, D.; Orikasa, T.; Xu, Q.; Okadame, H.; Nakamura, N.; Shiina, T. A review of life cycle assessment (LCA) on some food products. Journal of Food Engineering, v.90, p.1-10, 2009.

Ruviaro, C. F.; Gianezini, M.; Brandão F. S.; Winck, C. A.; Dewes, H. Life cycle assessment in Brazilian agriculture facing worldwide trends. Journal of Cleaner Production, v.28, p.9-24, 2012.

Seabra, J. E. A.; Macedo, I. C.; Chum, H. L.; Faroni, C. E; Sarto, C.A. Life cycle assessment of Brazilian sugarcane products: GHG emissions and energy use. Biofuels, Bioproducts and Biorefining, v.5, p.519-532, 2011.

Silva, V. P. da; Soares, S. R.; Alvarenga, R. A. F. Cradle to gate study of two differing Brazilian poultry production systems. In: International Conference on LCA in the Agri-Food Sector, 6, 2008. Zurich. Proceedings...Zurich: Agroscope Reckenholz-Tänikon Research Station ART, 2008, p.234-241.

Silva, V. P. da; Werf, H. M. G. V. D.; Spies, A.; Soares, S. R. Variability in environmental impacts of Brazilian soybean according to crop production and transport scenarios. Journal of Environmental Management, v.91, p.1831-1839, 2010.

Thorn, M. J.; Kraus, J. L.; Parker, D. R. Life-cycle assessement as sustainability management tool: Strengths, weaknesses, and other considerations. Enviromental Quality Management, v.20, p.1-10, 2011.

Valério, F. Environmental impacts of post-consumer material managements: Recycling, biological treatments, incineration. Waste Management, v.30, p.2354-2361, 2010. 